\title{
Solvation effects drive the selectivity in Diels-Alder reaction under hyperbaric conditions
}

\author{
Daniele Loco, ${ }^{1}$ Riccardo Spezia ${ }^{*, 1}$ François Cartier, ${ }^{1}$ Isabelle Chataigner*,1,2 \\ Jean-Philip Piquemal*,1,3 \\ ${ }^{1}$ Sorbonne Université, Laboratoire de Chimie Théorique, UMR 7616 CNRS, 75005 \\ Paris, France. \\ 2 Normandie Université, INSA Rouen, UNIROUEN, CNRS, COBRA laboratory, \\ F-76000 Rouen, France. \\ ${ }^{3}$ Institut Universitaire de France, 75005, Paris, France \\ Contacts: riccardo.spezia@sorbonne-universite.fr, \\ jean-philip.piquemal@sorbonne-universite.fr (SU) isabelle.chataigner@univ-rouen.fr \\ (Rouen)
}

March 9, 2020

\begin{abstract}
High pressure effects on the Diels-Alder reaction in condensed phase are investigated by means of theoretical methods, employing advanced multiscale modeling approaches based on physically grounded models. The simulations reveal how the increase of pressure from 1 to 10000 atm does not affect the stability of the reaction products, modifying the kinetics of the process by lowering considerably the transition state energy. The reaction profile at high pressure remarkably differs from that at $1 \mathrm{~atm}$, showing a submerged TS and a pre-TS structure lower in energy. The different solvation between endo and exo pre-TS is revealed as the driving force pushing the reaction toward a much higher preference for the endo product at high pressure.
\end{abstract}

The favourable effect of high pressure (HP) regimes on the kinetics of certain organic reactions is known for a while, ${ }^{1}$ notably for hindered substrates. HP indeed activates reactions unfeasible under conventional conditions, often increasing chemo-, regioand stereo- selectivities. ${ }^{[-4]}$ It also induces a significant reduction in energy costs by circumventing the continuous heating of the medium. As such, it can be considered as a "physical catalysis".

HP effect has been markedly studied on the DielsAlder cycloaddition,,$[6]$ a remarkable chemical transformation which still provides key solutions for the synthesis of (poly)cyclic molecules. The inertia of the components may limit its applicability, for steric or electronic reasons. The process is then usually activated by heating, using catalysts or changing solvents. Temperature increase often favors the reverse reaction, while catalysts may induce substrates and/or products degradation. Working under pressure is often an efficient alternative.

Cycloaddition reactions are characterized by very negative activation volumes $\left(\Delta \mathrm{V}^{\ddagger}=-20\right.$ to $\left.40 \mathrm{~cm}^{3} \cdot \mathrm{mol}^{-1}\right)^{[7}$ and their kinetics are usually accelerated under high pressure. One of the first and most emblematic examples is the use of furan as a diene in the synthesis of cantharidin by Dauben (see Figure 1, top). ${ }^{819}$ Since then, HP activation has shown its efficiency in performing $(4+2)$ cycloadditions involving sterically hindered $10-12]$ or electronically deactivated ${ }^{13}-16$ substrates for instance. This soft and eco-compatible activation mode has thus given access to different complex and functionalized structures, in the context of total syntheses for example. $\frac{17 / 18}{18}$ The positive influence of pressure on the diastereoselectivity of Diels-Alder cycloaddition reactions has been observed in many cases (see Figure 1. bottom). Generally, the more compact endo approach is favored by a HPs. 2|19|20 In some cases endo kinetic adducts are obtained under hyperbaric cycloaddition, while exo thermodynamic ones are generated by heating. 21

The activation observed in organic reactions in 


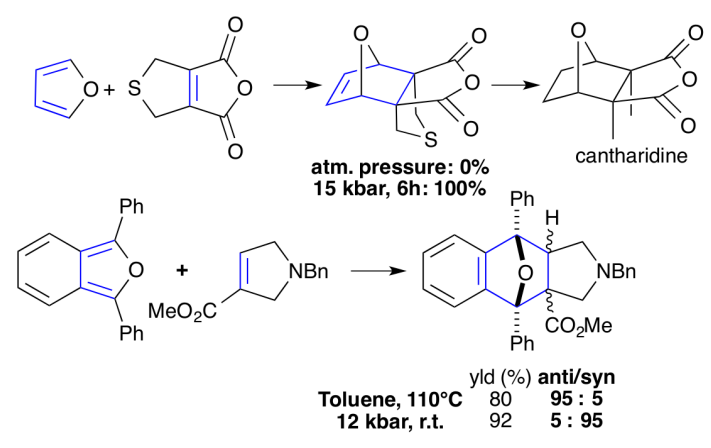

Figure 1: Examples of successful selectivity enhancement in organic chemistry reactions.

liquid phase is explained by the Evans-Polanyi equation, which connects a negative activation volume with the acceleration of the reaction with pressure increase. Based on such a theory, Ladanyi and Hynes studied pressure effects on rate constant of simple systems, finding an important dependence of the activation volume at very HP.22

Later, Dumas et al. have studied an aza-Michael addition via an atomistic model where the effect of pressure was included modifying the van der Waals radii. ${ }^{23}$ More recently, Hoffmann, Cammi and coworkers have studied the effect of pressure from a quantum chemistry approach coupled with an implicit solvation model on a set of organic reactions. ${ }^{24}$ The activation volumes was thus evaluated from electronic densities.

To get more insight into this phenonenon, we undertook a computational modeling study of pressure effects on a prototypical Diels-Alder reaction, between cyclopentadiene and acrylonitrile (see Figure 2.25

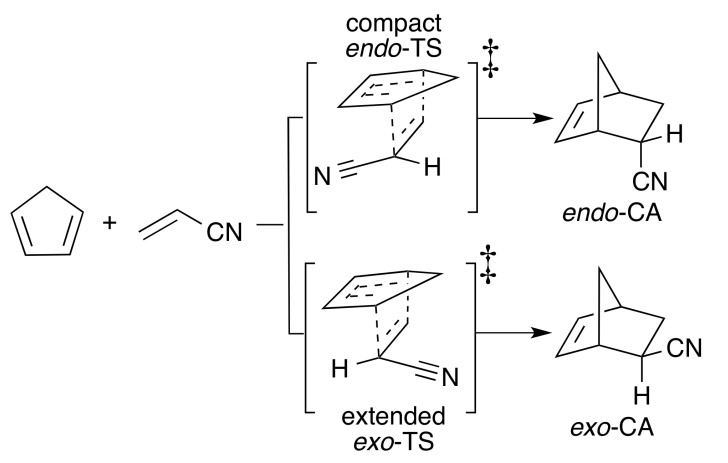

Figure 2: Model Diels-Alder reaction used in the present work. TS: transition state, CA: cycloadduct

Since the HP effect can modify the solute, the solvent and their interactions, we have adopted a physically grounded description of the solvent by means of an advanced classical polarizable force field $(\mathrm{FF}), 26$ coupled with a quantum chemistry method to treat the solute reacting species, which are then explicitly affected by the presence of the classical but atomistic solvent. This is what we define a polarizable variational $\mathrm{QM} / \mathrm{MM}$ method.27/29

To sample the large number of solute-solvent configurations needed to extract meaningful energies and properties from such atomistic models we resorted to a sequential approach: first performing full FF based molecular dynamics to have equilibrated solvent configurations at the needed temperature and high/low pressures, then including the quantum treatment of the solute in the structure sampling and performing shorter QM/MM MDs, starting from different points of the full FF-based trajectories.

The solute molecules are described within the the density functional theory (DFT) approximation, with the M06-2X functional ${ }^{30}$ and a 3-21G atomic basis set. A detailed discussion for this choice is reported in the Supporting Informations (SI, Section S1). Note that it is not uncommon that a small basis set can provide results better than a larger one: $\underline{31}$ convergence in basis set sometimes occur only for a very large one, which would be then inadequate to perform QM/MM MDs.

During the polarizable MDs the solvent has been modeled with the AMOEBA FF, which performs correctly at different pressures without any ad hoc parametrization. ${ }^{32}$ Solute was solvated by 603 $\mathrm{CH}_{2} \mathrm{Cl}_{2}$ molecules using an AMOEBA potential recently developed for organic molecules. ${ }^{33}$ The solvent was first equilibrated at room temperature and at two different pressures (low, 1 atm and high, $10 \mathrm{katm}$ ) fixing the solutes in their QM optimized geometry and running about 2 ns polarizable MD simulations using the Bussi-Parrinello algorithm to keep an NPT ensemble. 34

This procedure was performed for each chemical species relevant to describe the reaction pathway, and thus placed as solute inside the $\mathrm{CH}_{2} \mathrm{Cl}_{2}$ box of solvent. These structures represent: (i) the reactants, (ii) transition states (TS), endo and exo approaches and (iii) cycloadducts (CA), endo and exo diastereomers. Intermediates between reactants and TS were also considered (vide infra) and named here (iv) pre-TS. These structures were taken from the intrinsic reaction coordinate (IRC) which connects reactants with TS (see SI for more details). The solvent equilibration was performed for the two different pressure regimes. 
After an equilibration time of less than $10 \mathrm{ps}$ the systems reached the equilibrium density at the two different pressures, namely $1.419(3)$ and $1.776(4) \mathrm{g} / \mathrm{mL}$. These values are independent on the chemical nature of the solutes. In Figure 3 the density time series at the beginning of the classical polarizable trajectories are reported, showing the differences between low and high pressure simulations, for which also different simulation box sizes were obtained.

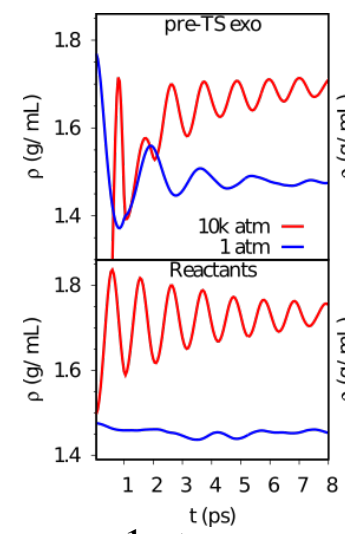

$1 \mathrm{~atm}$

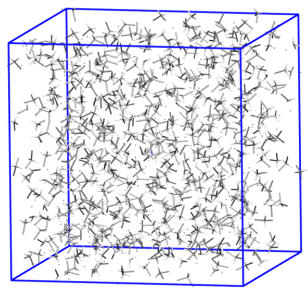

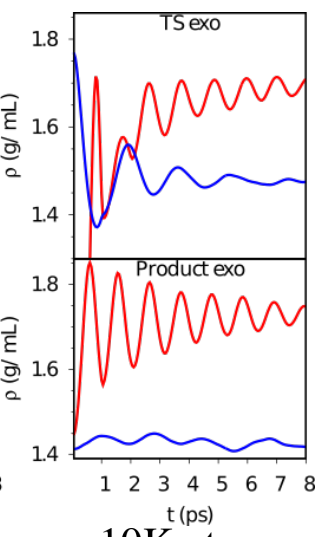

$10 \mathrm{~K}$ atm

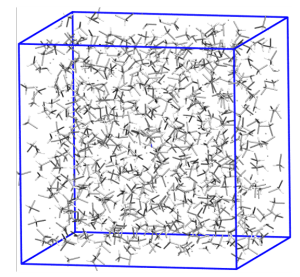

Figure 3: Top) Solution density's time-series along the initial part of the full-FF equilibration dynamics run for pre-TSs, TSs, cycloadducts and reactants in exo configuration, in $\mathrm{CH}_{2} \mathrm{Cl}_{2}$ solution. In red are reported the densities for the $10 \mathrm{katm}$ MDs, while those for 1 atm MDs are in blue. Bottom) Shrinking of the MD simulation box due to the pressure increase. The cubic boxes reported are taken from two trajectories of the same solvated system at 1 and $10 \mathrm{katm}$, with a box side length of 39 and $36 \AA$ respectively

Finally, the solute-solvent interaction was refined via a variational formulation of hybrid polarizable $\mathrm{QM} / \mathrm{MM}$, accounting for the mutual polarization between the classical and the quantum subsystems. The forces needed for QM/MM dynamics were consistently derived from the energies computed in this way. $27 \sqrt[29135]{2}$ This approach was used for the first time here to investigate pressure effects on a chemical reaction.

Once the solvation was equilibrated via polar- izable $\mathrm{MD}, \mathrm{QM} / \mathrm{MM}$ simulations were performed, either running few picoseconds QM/MM MD or single point $\mathrm{QM} / \mathrm{MM}$ energy calculations (see Section S3 of SI), using structures sampled from the full-FF based polarizable MDs. The Tinker-HP package ${ }^{36}$ coupled with a development version of Gaussian09 was used for all the calculations. $\frac{37}{37}$

The reaction energy profiles were thus obtained extracting average energies from the simulations at different pressures, setting at zero the energy of the reactants at both $1 \mathrm{~atm}$ and $10 \mathrm{katm}$. Such relative energies are reported in Figure 4 where we also draw the structures of important geometries along the reaction pathway.

In the $1 \mathrm{~atm}$ reaction pathway, the TS was found to be 11 and $18 \mathrm{kcal} / \mathrm{mol}$ higher than reactants for endo and exo approaches, respectively (with a statistical uncertainty of $1 \mathrm{kcal} / \mathrm{mol}$ for these and further energy values). Products were exothermic of 38 and $39 \mathrm{kcal} / \mathrm{mol}$ for endo and exo pathways. Note that for such a reaction a very small difference between the two stereomers is expected. ${ }^{[38}$ Finally, at $1 \mathrm{~atm}$ the pre-TS has an energy lying in between the reactants and the TS, as in the IRC calculations from which the structures were extracted.

At $10 \mathrm{katm}$ the situation is very different instead: the TS was found to be energetically more stable than the reactants. Interestingly, the pre-TS structures are now more stable than the corresponding TS. However, while for the exo reaction, a barrier of about $9 \mathrm{kcal} / \mathrm{mol}$ was calculated between the preTS and the TS, for the endo reaction it was found to be almost zero $(1 \mathrm{kcal} / \mathrm{mol}$ which lies into the uncertainty). This means that the rate constant in the case of the endo approach is almost under diffusion control and much faster than the exo one. Finally, products were similar in energy with a slight preference for the endo diastereomer.

Results show that the reaction is indeed kinetically favoured by a pressure increase. For the exo approach, an energy barrier of $18 \mathrm{kcal} / \mathrm{mol}$ at ambient pressure translates into a $9 \mathrm{kcal} / \mathrm{mol}$ one at HP; for endo approach, the difference is even bigger: the $11 \mathrm{kcal} / \mathrm{mol}$ barrier observed at ambient pressure almost vanishes at HP and the kinetics is then diffusion controlled. Finally, the resulting endo cycloadduct is favoured (due to kinetic control) with respect to exo, as experimentally expected.

In our microscopic description of the system, increasing the pressure triggers a compact packing of the molecules. As a consequence, the attractive interactions with the solvent molecules increase (see 

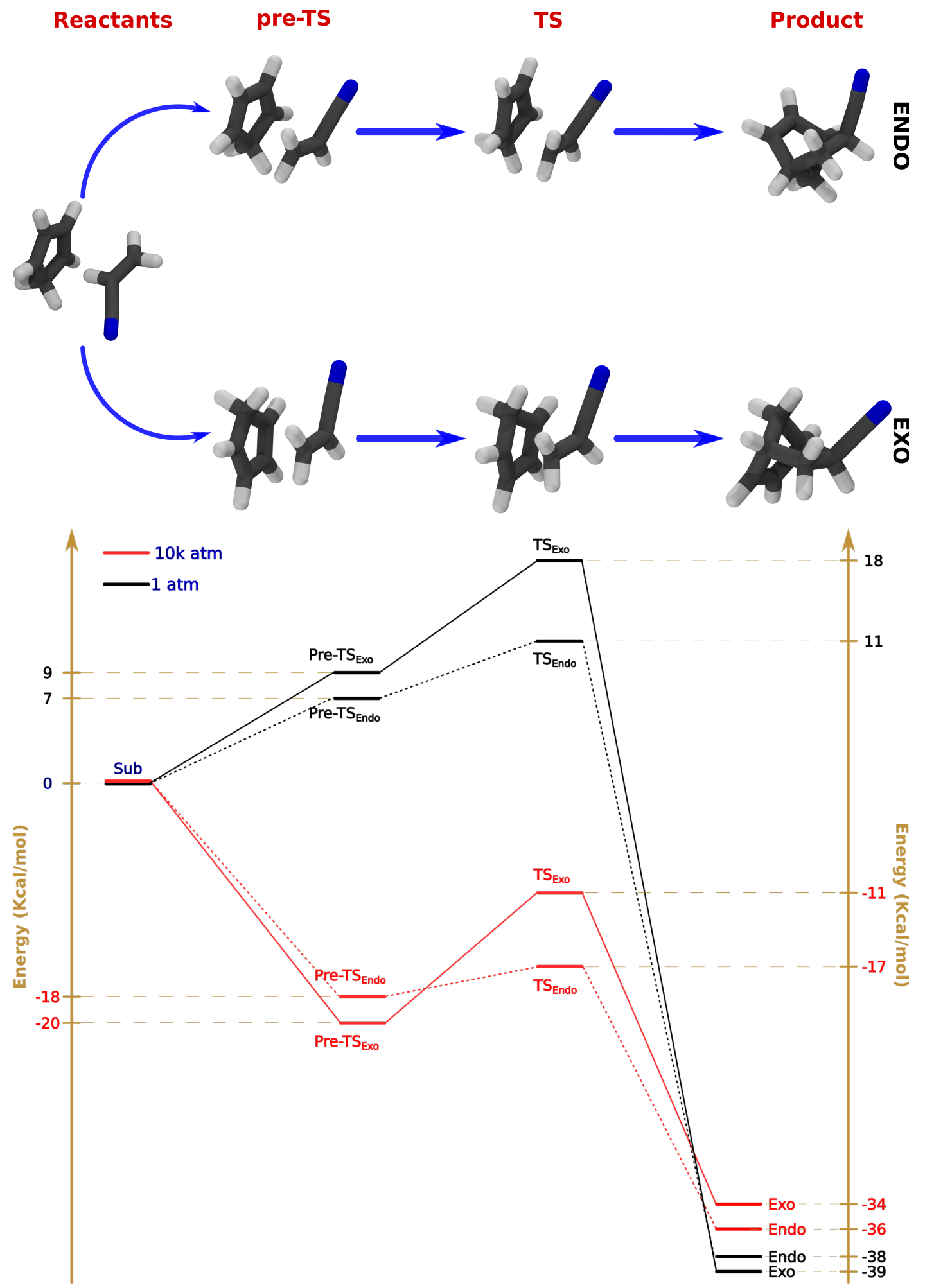

Figure 4: Top) Structures of the different molecular species involved in the reaction. Bottom) Energy diagram as from $\mathrm{QM} / \mathrm{MM}$ simulations 
Table S2 of SI for the absolute energetics). In this respect, the use of polarizable $\mathrm{FFs}^{26}$ is crucial: the polarization variation with the pressure ensures a large part of this stabilization (see Table S2 of SI). Only thanks to the fluctuations of their induced dipole, the classical solvent molecules are allowed to respond to the different pressure regimes and readjust during the compaction of the molecular packing.

On one hand, the extended exo-stereomers are more exposed to the solvent molecules than the compact endo ones. This effect is even greater in the pre-TS where the substrates are further apart: the stabilizing interactions induced by the solvent under HP show their maximum effect for the exo pre-TS, which is more stable than the endo one by $2 \mathrm{kcal} / \mathrm{mol}$. On the other hand the TS endo is generally considered to be more favoured than the TS exo, due to the attractive secondary orbital interactions between the $\mathrm{p}$ orbitals that are not directly involved in the formation of the new $\sigma$ bonds, according to the famous (even if still debated) proposition by Woodward and Hoffman 39 . As a net result of these two antagonistic phenomena, the endo TS is almost barrier less, while the exo one features an energy activation of $9 \mathrm{kcal} / \mathrm{mol}$. Thus, the solvation effect explains the greater preference for the endo cycloadduct formation under HP.

We connect our results to the commonly accepted picture offered by the concept of activation volume. Following the approach used by Cammi and co-workers, $\stackrel{40}{a}$ we compute molar volumes from $\mathrm{QM} / \mathrm{MM}$ electron densities at the two different pressures, using them to compute activation and reaction volumes. As shown in Table 1, for each species the activation volume does not depend neither on the pressure, nor on the stereochemistry. This means that we are in the linear regime of pressure dependence, confirming experimental data. $\frac{\sqrt{3}}{}$ Furthermore, the reaction volumes are similar, showing the high similarity between TS and products as expected for such a late transition state Diels-Alder reaction.

The pre-TS were found to be crucial in differenciating the kinetics between the exo and endo approaches at HP. This is in line with the different activation volumes calculated when the pre-TSs were considered as reactants (see Table 1). The exo pre-TS volume is indeed similar to the reactants one, while the endo pre-TS volume resembles more the corresponding TS at HP (the absolute volume of each structure is reported in Fig. S4 of SI).

In conclusion, we propose here that the solvation
Table 1: Activation volume $\Delta \mathrm{V}^{\ddagger}$ and reaction volume $\Delta \mathrm{V}^{\text {react }}$ for the reaction under investigation computed from the SCF QM/MM electron density with Monte-Carlo integration by Gaussian 09 40 . The values, with associated $95 \%$ confidence interval, are reported in $\mathrm{cm}^{3} / \mathrm{mol}$. The molecular volume is defined as the volume inside a contour $\rho$ (electron $/$ bohr $^{3}$ ) density set at the Gaussian09 default value

\begin{tabular}{|c|c|c|c|c|}
\hline & \multicolumn{2}{|c|}{$\Delta \mathrm{V}^{\ddagger}$} & \multicolumn{2}{|c|}{$\Delta \mathrm{V}^{\text {react }}$} \\
\hline & Endo & Exo & Endo & Exo \\
\hline \multirow{2}{*}{$1 \mathrm{~atm}$} & -8.65 & -10.09 & -10.76 & -10.24 \\
\hline & \pm 2.60 & \pm 3.60 & \pm 0.60 & \pm 0.60 \\
\hline \multirow{4}{*}{$10 \mathrm{k} \mathrm{atm}$} & -9.00 & -8.94 & $-11,12$ & $-10,594$ \\
\hline & \pm 3.20 & \pm 3.60 & \pm 0.60 & \pm 0.60 \\
\hline & \multicolumn{2}{|c|}{$\Delta \mathrm{V}_{\mathrm{pre}-\mathrm{TS}}$} & & \\
\hline & Endo & Exo & & \\
\hline \multirow{2}{*}{$1 \mathrm{~atm}$} & -6.34 & -3.71 & & \\
\hline & \pm 4.10 & \pm 4.80 & & \\
\hline \multirow{2}{*}{$10 \mathrm{k}$ atm } & -4.40 & -7.40 & & \\
\hline & \pm 4.10 & \pm 4.80 & & \\
\hline
\end{tabular}

plays a fundamental role on the pressure effect on this Diels-Alder prototypical cycloaddition. Under $\mathrm{HP}$, the efficient solvation of the TSs lowers the energy barriers and explain the acceleration of the process. In addition to this phenomenon, the exo preTS arrangement, more exposed to solvent molecules, is favorably solvated under HP and more stabilized than the endo one, making the passage to the corresponding TS more challenging. In the meantime, the compact endo TS remains favored thanks to its attractive secondary orbital interactions. These two parallel phenomena explain the enhancement of the diastereoselectivity and the greater endo preference for Diels-Alder cycloaddition under HP. Note that this interpretation is somehow in line with the work by Houk and Bickelhaupt, showing that a distortion/interaction model is especially fruitful for explaining cycloaddition reactivity. 41 The QM/MM method developed in our group shows how pressure effects in the condensed phase can be modeled for an archetypal Diels-Alder reaction. The original approach proposed will certainly find other applications, not only for transformations under HP, but also to further investigate the crucial role of solvents/additives in organic reactions. 


\section{Acknowledgements}

This work has received funding from the European Research Council (ERC) under the European Union's Horizon 2020 research and innovation program (grant agreement No 810367), project EMC2. Computations have been performed at GENCI on the Occigen machine (CINES, Montpellier, France) on grant no A0070707671.

\section{Keywords}

Cycloaddition - High-pressure chemistry - Molecular Dynamics · QM/MM simulations · Reaction mechanism

\section{References}

[1] W. C. Röntgen, Ann. Phys. Chem. 1892, 281, 98-107.

[2] W. J. le Noble, H. Kelm, Angew. Chem. Int. Ed. Engl. 1980, 19, 841-856.

[3] R. van Eldik, F. Klärner, High Pressure Chemistry: Synthetic, Mechanistic, and Supercritical Applications, Wiley, 2002.

[4] I. Chataigner, J. Maddaluno in Activation methods: Sonochemistry and High Pressure (Ed.: C. J. Goddard, M. Malacria), Wiley, 2019, pp. 95-149.

[5] K. Matsumoto, A. Sera, Synthesis 1985, 1985, 999-1027.

[6] K. Matsumoto, H. Hamana, H. Iida, Helv. Chim. Act. 2005, 88, 2033-2234.

[7] A. Drljaca, C. D. Hubbard, R. van Eldik, T. Asano, M. V. Basilevsky, W. J. le Noble, Chem. Rev. 1998, 98, 2167-2290.

[8] W. G. Dauben, C. R. Kessel, K. H. Takemura, J. Am. Chem. Soc. 1980, 102, 6893-6894.

[9] C. Petrier, J. L. Luche, J. Org. Chem. 1985, 50, 910-912.

[10] B. Gacem, G. Jenner, J. Phys. Org. Chem. 2004, 17, 221-225.

[11] G. Jenner, Tetrahedron 2005, 61, 3621-3635.
[12] R. Baker, D. L. Selwood, C. J. Swain, N. M. H. Webster, J. Hirshfield, J. Chem. Soc. Perkin Trans. 1 1988, 471-480.

[13] R. W. Aben, J. Keijsers, B. Hams, C. G. Kruse, H. W. Scheeren, Tetrahedron Lett. 1994, 35, 1299-1300.

[14] K. Kumamoto, I. Fukada, H. Kotsuki, Angew. Chem. Int. Ed. 2004, 43, 2015-2017.

[15] A. Chrétien, I. Chataigner, S. R. Piettre, Chem. Commun. 2005, 1351-1353.

[16] N. Chopin, H. Gérard, I. Chataigner, S. R. Piettre, J. Org. Chem. 2009, 74, 1237-1246.

[17] C. L. Hugelshofer, T. Magauer, Synthesis 2014, 46, 1279-1296.

[18] M. Uroos, P. Pitt, L. M. Harwood, W. Lewis, A. J. Blake, C. J. Hayes, Org. Biomol. Chem. 2017, 15, 8523-8528.

[19] M. Buback, J. Abeln, T. Hübsch, C. Ott, L. F. Tietze, Liebigs Ann. 1995, 1995, 9-11.

[20] L. F. Tietze, M. Henrich, A. Niklaus, M. Buback, Chem. Eur. J. 1999, 5, 297-304.

[21] N. Pichon, A. Harrison-Marchand, P. Mailliet, J. Maddaluno, J. Org. Chem. 2004, 69, 72207227.

[22] B. M. Ladanyi, J. T. Hynes, J. Am. Chem. Soc. 1986, 108, 585-593.

[23] F. Dumas, C. Fressigné, J. Langlet, C. GiessnerPrettre, J. Org. Chem. 1999, 64, 4725-4732.

[24] B. Chen, R. Hoffmann, R. Cammi, Angew. Chem. Int. Ed. 2017, 56, 11126-11142.

[25] K.Nakagawa, Y.Ishii, M.Ogawa, Tetrahedron 1976, 32, 1427-1429.

[26] J. Melcr, J.-P. Piquemal, Front. Biosci. 2019, $6,143$.

[27] D. Loco, Polack, S. Caprasecca, L. Lagardère, F. Lipparini, J.-P. Piquemal, B. Mennucci, $J$. Chem. Theory Comput. 2016, 12, 3654-3661.

[28] D. Loco, L. Lagardère, S. Caprasecca, F. Lipparini, B. Mennucci, J.-P. Piquemal, J. Chem. Theory Comput. 2017, 13, 4025-4033. 
[29] D. Loco, L. Lagardère, G. A. Cisneros, G. Scalmani, M. Frisch, F. Lipparini, B. Mennucci, J.-P. Piquemal, Chem. Sci. 2019, 10, 72007211.

[30] Y.Zhao, D.G.Truhlar, Theor. Chem. Acc. 2008, 120, 215-241.

[31] A.Martin-Somer, M.-P.Gaigeot, M.Yanez, R.Spezia, Phys. Chem. Chem. Phys. 2014, 16, 14813-14825.

[32] P. Ren, J. W. Ponder, J. Phys. Chem. B 2004, 108, 13427-13437.

[33] X. Mu, Q. Wang, L.-P. Wang, S. D. Fried, J.-P. Piquemal, K. N. Dalby, P. Ren, The Journal of Physical Chemistry B 2014, 118, 6456-6465.

[34] G. Bussi, D. Donadio, M. Parrinello, J. Chem. Phys. 2007, 126, 014101.

[35] F. Lipparini, J. Chem. Theory Comput. 2019, 15, 4312-4317.

[36] L. Lagardère, L.-H. Jolly, F. Lipparini, F. Aviat, B. Stamm, Z. F. Jing, M. Harger, H. Torabifard, G. A. Cisneros, M. J. Schnieders, N. Gresh, Y. Maday, P. Y. Ren, J. W. Ponder, J.-P. Piquemal, Chem. Sci. 2018, 9, 956-972.

[37] M. J. Frisch et al, Gaussian Development Version, Revision H.36, Gaussian Inc. Wallingford CT 2010.

[38] O. Larranaga, A. de Cozar, ChemistryOpen 2019, 8, 49-57.

[39] C. S. Wannere, A. Paul, R. Herges, K. N. Houk, H. F. Schaefer III, P. Von Ragué Schleyer, J. Comput. Chem. 2007, 28, 344-361.

[40] T. Yang, R. Fukuda, R. Cammi, M. Ehara, J. Phys. Chem A 2017, 121, 4363-371.

[41] F. M. Bickelhaupt, K. N. Houk, Angew. Chem. Int. Ed. 2017, 56, 10070-10086. 